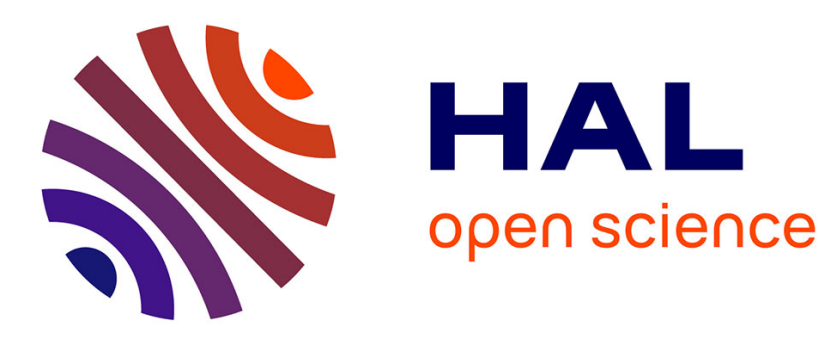

\title{
Electron transport through a gold-bisthiolterthiophene-gold junction
}

Christophe Kergueris, Jean-Philippe Bourgoin, Serge Palacin, Daniel Estève, Cristian Urbina, M. Magoga, Christian Joachim

\section{- To cite this version:}

Christophe Kergueris, Jean-Philippe Bourgoin, Serge Palacin, Daniel Estève, Cristian Urbina, et al.. Electron transport through a gold-bisthiolterthiophene-gold junction. AIP Conference Proceedings, 1999, 486, pp.421-426. 10.1063/1.59822 . cea-01057028

\section{HAL Id: cea-01057028 https://hal-cea.archives-ouvertes.fr/cea-01057028}

Submitted on 18 Apr 2018

HAL is a multi-disciplinary open access archive for the deposit and dissemination of scientific research documents, whether they are published or not. The documents may come from teaching and research institutions in France or abroad, or from public or private research centers.
L'archive ouverte pluridisciplinaire HAL, est destinée au dépôt et à la diffusion de documents scientifiques de niveau recherche, publiés ou non, émanant des établissements d'enseignement et de recherche français ou étrangers, des laboratoires publics ou privés. 


\section{Electron transport through a gold-bisthiolterthiophene-gold}

\section{junction}

C. Kergueris, J.-P. Bourgoin, S. Palacin, D. Esteve, C. Urbina, M. Magoga, and C. Joachim

Citation: AIP Conference Proceedings 486, 421 (1999); doi: 10.1063/1.59822

View online: https://doi.org/10.1063/1.59822

View Table of Contents: http://aip.scitation.org/toc/apc/486/1

Published by the American Institute of Physics 


\title{
Electron transport through a gold-bisthiolterthiophene-gold junction
}

\author{
C. Kergueris ${ }^{a}$, J.-P. Bourgoin ${ }^{a 1}$, S. Palacin ${ }^{a}$, D. Esteve ${ }^{b}$, C. Urbina ${ }^{b}$, \\ M. Magoga ${ }^{c}$, C. Joachim ${ }^{c}$
}

a. Service de Chimie Moléculaire, b. Service de Physique de l'État Condensé, CEA-Saclay, 91191 Gif-sur-Yvette Cedex, France

c. CEMES-CNRS BP 4347, 31055 Toulouse Cedex, France

\begin{abstract}
Molecules of bisthiolterthiophene have been adsorbed on the two facing gold electrodes of a mechanically controllable break junction in order to form metalmolecule(s)-metal junctions. Current-voltage (I-V) characteristics have been recorded at room temperature. Zero bias conductances were measured in the 10-100 nS range and different kinds of non-linear I-V curves with step-like features were reproducibly obtained. A scattering model is used to interpret the experimental conductances as a function of the metal-molecule coupling strength.
\end{abstract}

\section{INTRODUCTION}

The experimental investigation of the transport properties of a single or a very few molecules contacted with two metallic electrodes has seen significant advances in the last few years. Three different strategies have been used, so far, to solve the problem of connecting a molecule to two facing metallic electrodes. This includes: i) using a Scanning Tunneling Microscope (STM) to contact a molecule adsorbed on a planar substrate [1-5], ii) using planar electron-beam lithographed electrodes separated by a small gap bridged by the molecule $[6,7]$, iii)placing the molecule in the gap of a mechanical break junction (MCB) [8-11].

In the present paper, we describe the use of gold MCB junctions to investigate the electronic transport properties of 2.5"-bis(acetylthio)-5.2'5'.2" -terthienyl (T3) molecules. We present different types of I-V characteristics and compare the experimental zero bias conductance with the value calculated by means of a scattering model based on an extended Hückel description of the system. In particular, we study the influence of the metal-molecule coupling strength on the results of the calculation.

1) Corresponding author. E-mail: jbourgoin@cea.fr

CP486, Electronic Properties of Novel Materials - Science and Technology of Molecular Nanostructures, edited by H. Kuzmany, J. Fink, M. Mehring, and S. Roth

(C) 1999 American Institute of Physics 1-56396-900-9/99/\$15.00 


\section{EXPERIMENTAL RESULTS}

The experimental details concerning the synthesis of the T3 (Fig. 1) molecule, the fabrication of the suspended gold microbridges by e-beam lithography and the electrical measurement setup have been described in ref [11]. The elastic substrate supporting the microbridge was mounted in a three point bending configuration. It was bent by pushing in its center with a driving rod actuated by a coarse adjustment screw until the resistance becomes infinite, which indicated that the bridge was broken. The molecules were immediately self-assembled onto the freshly broken electrodes by immersion of the broken junction in a droplet of a solvent containing the molecules (a $5.10^{-4} \mathrm{~mol} \mathrm{l}^{-1}$ solution of $\mathbf{T} 3$ in trichloro-1,1,1-ethane was used; 0.1 $\%$ of dimethylaminoethanol were added to the solution $1 \mathrm{~min}$ before the experiment for deprotection). Then the solvent was evaporated and Argon was continuously flushed through the sealed box during the experiment. The experimental conditions (short time equilibration with the solution and Ar flow) were chosen to hinder as much as possible the formation of di- or polydi-sulfides. In a final step, the bridge gap was reduced using a piezoelectric fine adjustment of the driving rod until current detection.

Various control experiments have been performed including measurements on Auair-Au junctions, for which the exponential variation of conductance with distance was checked, on Au-air-Au junctions after 1 min immersion in the pure solvent and on Au-dodecanethiol(s)-Au junctions: the corresponding I-V curves were featureless with a linear behaviour at low bias. [10] The results were markedly different when T3 molecules were introduced in the gap of the junction. During a typical experiment on $\mathrm{Au}-\mathrm{T} 3-\mathrm{Au}$ junctions, stability periods with a duration $1-20 \mathrm{~min}$ alternated with instability periods generally lasting a few minutes. This behavior was always observed on the four samples that have been measured. Although different I-V characteristics could be observed, the reproducible ones were of one

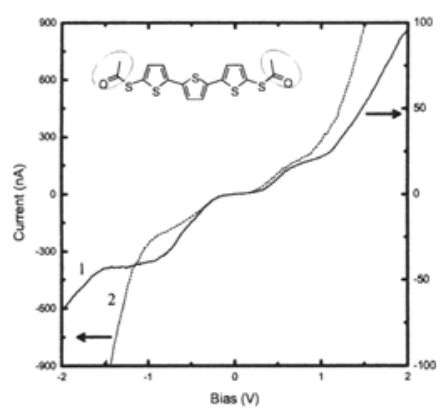

FIGURE 1. The T3 molecule investigated (the circled protective groups are removed prior to assembly) and typical (1) asymmetric (solid line) and (2) symmetric (dashed line) I-V curves recorded at room temperature for gold-T3-gold junctions. Both curves were obtained by averaging over 5 voltage sweeps. 
of the two types of I-Vs shown in Fig.1. Asymmetric I-Vs ressembling type (1) were more often observed and more stable than symmetric ones of type (2).The measured zero bias conductance of type (1) junctions is of the order of $10 \mathrm{nS}$. The asymmetric I-V characteristic is non-linear with step-like features, and the current increases linearly at large voltage. The measured zero bias conductance of type (2) junctions is larger, about $80 \mathrm{nS}$. The symmetric $\mathrm{I}-\mathrm{V}$ characteristic is also non-linear with smaller step-like features. At $V \geq 1 \mathrm{~V}$, the current rises faster than linearly with $V$.

\section{DISCUSSION}

Several models have been proposed to explain the transport mechanism is this kind of metal-molecule-metal. They can be classified into sequential and coherent models. In the former, each electron (hole) transported from one metallic electrode to the other one temporarily charges negatively (positively) the molecule which is considered to be weakly coupled to the electrodes through tunnel barriers. In the latter the tunneling electrons never localize on the molecule which is considered to be strongly coupled to the electrodes. It should be noted that a more realistic model should consider at the same time coherent and sequential transport. In what follows, we use a coherent model, the Electron Scattering Quantum Chemistry (ESQC) technique, $[12,13]$ that proved successful in interpreting metal$\mathrm{C}_{60}$-metal junction measurements, [14] to calculate the zero bias conductance of gold-T3-gold junctions and compare them with the experimental results. This technique treats the molecule as a defect which breaks the translational invariance of the metal, and therefore scatters incident electrons. In its present implementation, the ESQC technique ignores both the electron-electron and electron-phonon interactions and neglects charging effects. It assumes that the scattering is elastic because

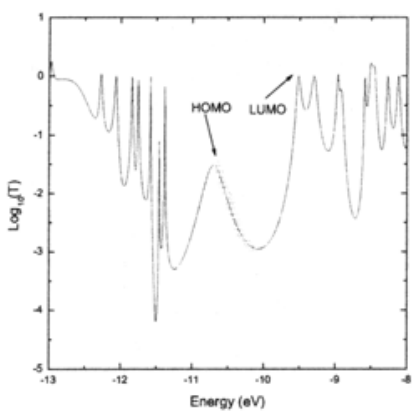

FIGURE 2. The transmission function of the gold-T3-gold junction calculated by the ESQC technique assuming a Au-S distance of $1.95 \AA$ at both end of the molecule. HOMO and LUMO refer to the gap of T3 adsorbed on the two electrodes. The energy scale reference is arbitrary. The calculated position of the Fermi level of the electrodes falls in the hatched area. 
for molecule of small length, the tunneling time is shorter than the intramolecular relaxation times. An extended Hückel model is used to build up the matrix representation of the multichannel scattering Hamiltonian taking into account the complete chemical description of the electrodes and of the molecule. The calculated multichannel transmission coefficient $T(E)$ of an electron at a given energy $E$ is shown in Fig. 2. For this calculation, we made the assumption that a single molecule is involved, its geometry being optimized at the AM1 level in a forced planar conformation. [15] The linear conductance $G$ of the metal-molecule-metal junction is then determined using the Landauer formula [16],

$$
G=\frac{2 e^{2}}{h} T\left(E_{F}\right)
$$

where $E_{f}$ is the Fermi level of the electrodes.

The prediction for $G$ thus depends on the estimated position of the electronic spectrum relatively to the Fermi energy $E_{F}$, on the exact conformation of the molecule in the junction and of the coupling of the molecule to the electrodes. As shown in Ref [11], the position of the Fermi level depends on the exact amount of charge transferred upon formation of the $\mathrm{Au}-\mathrm{S}$ bond. It can be reasonably estimated in the $\left[\mathrm{E}_{\text {HOMO }}, \mathrm{E}_{\text {HOMO }}+0.7 \mathrm{eV}\right]$ range. The strength of coupling is determined by the length of the Au-S bonds. The $\mathrm{S}$ atoms were assumed to be adsorbed in a hollow site of the gold surface. A bond length of $1.905 \AA$ was used in the present calculation [17]. This is the shortest distance we found in the literature. It thus provides an upper bound for the coupling strength. Assuming a symmetric coupling at both ends of the molecule, the calculated conductances for $E_{F}-E_{H O M O}=$ 0.6 (midgap), 0.2 and $0 \mathrm{eV}$ are $87,585,2306 \mathrm{nS}$, respectively. Although the order of magnitude of these values is comparable with the measured one $G \simeq 10-80 \mathrm{nS}$, the discrepancy indicates that the coupling of the molecule to the electrodes is smaller than estimated. In order to study the influence of the strength of coupling on the calculated spectrum, we performed systematic calculations, where we varied the distance between the gold electrodes and the molecules, first keeping a symmetric coupling at both ends of the molecule, and second keeping a fixed $1.905 \AA$ at one end and varying the second distance. The results are shown in Fig.3. These results can be explained as follows. First, the smaller the coupling (symmetric or not) the thinner the resonance peaks. Second, as expected from a simple analogy with double barrier resonnant tunneling devices, the height of the peaks decreases for an asymmetric coupling not for a symmetric one. Third, the transmission is dominated by the weakest coupling. Fourth, the resonance corresponding to the HOMO is strongly affected by the modification of coupling. This is due to the fact that it corresponds to the superposition of four peaks: two very small ones corresponding to two highly localized orbitals that are not efficient for the transport and two dominant ones, close in energy, that give rise to a destructive interference. When the coupling is modified, the width of the two dominant peaks decreases and the interference as well. Consequently, if the Fermi Level is located at the HOMO, a reduction of coupling should correspond to an increase of conductance. The curves 
are relatively insensitive to the variation of coupling for Au-S distances up to $3 \AA$, but strongly modified for longer Au-S distances. As a consequence of the reduction of the coupling strength, the value of the conductance calculated assuming the Fermi level at midgap decreases. For example, for a symmetric(resp. asymmetric) coupling, the zero bias conductance reduces to $20 \mathrm{nS}(48 \mathrm{nS})$ for $\mathrm{d}(\mathrm{Au}, \mathrm{S})=3 \AA$ and $1.5 \mathrm{nS}(12 \mathrm{nS})$ for $\mathrm{d}(\mathrm{Au}, \mathrm{S})=4 \AA$. These results show i)that a reduction of coupling allows to get a more quantitative agreement between experiment and theory, except if the Fermi Level of the electrodes is very close to the HOMO and ii)that rather large values of Au-S bond lengths (i.e. small couplings)have to be used to obtain a good agreement between experiment and theory. This remark agrees with with the interpretation of the results of ref [8] by Emberly and Kirckzenow [18].

\section{CONCLUSION}

In this paper, we have investigated the transport properties of molecules of 2,5 "bis(acetylthio)-5, 2', 5', 2" -terthienyl self-assembled in the adjustable gap of a metallic break junction. We have observed that the I-V characteristics recorded at room temperature are not always symmetric with respect to the polarity of the applied bias and show two different regimes: a linear regime at low bias $V<0.1 \mathrm{~V}$ and
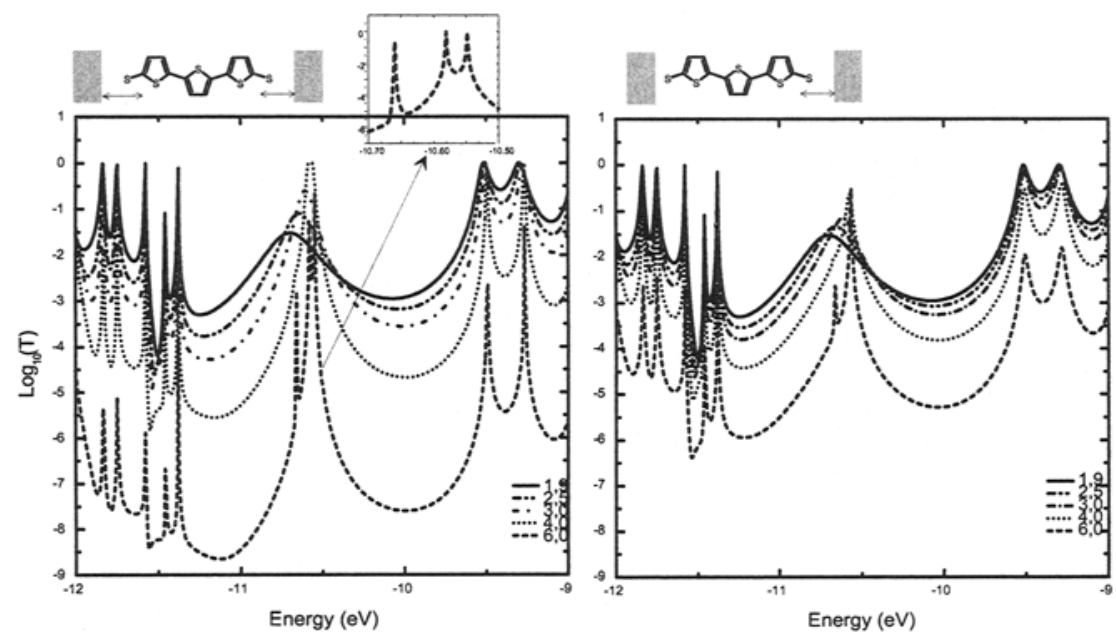

FIGURE 3. Transmission functions of the gold-T3-gold junction calculated by the ESQC technique as a function of coupling strength (symmetric: left; asymmetric: right) The strength of coupling is fixed by the Au-S distance (number in $\AA$ at the bottom right of each plot). The inset of the left figure is a close-up view of the peaks of the curve corresponding to $d(A u, S)=6 \AA$ calculated with a higher number of points. It shows that the reason why the peaks do not reach unity in the main graph is due to a lack of numerical accuracy. 
a highly non-linear regime with step-like features at higher voltage. The order of magnitude of the measured zero bias conductance is comparable to the theoretical calculation made with the ESQC technique assuming a single molecule in the gap of the break junction. This indicates that these experiments likely involve a very few molecules.

\section{REFERENCES}

1. Joachim C. and Gimzewski J. K. Chem. Phys. Lett. 265, 353 (1997).

2. Datta S, Tian W, Hong S, Reifenberger R, Henderson J I, Kubiak C P Phys. Rev. Lett. 792530 (1997)

3. Bumm L.A.,Arnold J.J.,Cygan M.T., Dunbar T.D., Burgin T.P.,Jones L., Allara D.L., Tour J.M., Weiss P.S. Science 271, 1705 (1996).

4. Dhirani A., Lin P.-H., Guyot-Sionnest P.,Zehner R.W. and Sita L.R. J. Chem. Phys. 106, 5249 (1997).

5. Rosink J.J.W.M., Blauw M.A., Geerligs L.J., Van der Drift E. Rousseeuw B.A.C., Radelaar S., Optical Mat. 9416 (1998)

6. Tans S.J., Devoret M.H., Dai H., Thess A., Smalley R.E.,Geerligs L.J. and Dekker C. Nature. 386, 474 (1997).

7. Ebbesen T.W., Lezec H.J., Hiura H., Bennett J.W., Ghaemi H.F. and Thio T. Nature. 382, 54 (1996).

8. M. A. Reed et al, Science 278, 252 (1997).

9. Muller C.J, Vleeming B.J., Reed M.A., Lambda J.J.S., Hara R., JonesII L, and Tour J.M.Nanotechnology 7, 409 (1996).

10. Kergueris C., Bourgoin J.P., Palacin S., Nanotechnology 10, 8 (1999)

11. Kergueris C., Bourgoin J.P., Palacin S., Estève D., Urbina C., Magoga M., Joachim C., Phys. Rev. B 59 (1999)

12. Sautet P. and Joachim C., Chem. Phys. Lett. 185, 23 (1989).

13. Joachim C. and Vinuesa J.F., Europhys. Lett. 33, 635 (1996).

14. Joachim C., Gimzewski J.K., Schlitter R.R. and Chavy C., Phys. Rev. Lett. 74, 2102 (1995).

15. For this calculation we used the following $3 s, 3 p$ and $3 d$ parameters for the $S$ atoms (S. Alvarez, Extended Hückel parameters, Barcelona University (1989)): $H_{i i}=-20$ $\mathrm{eV}, \xi_{i 1}=2.122, H_{i i}=-11.1 \mathrm{eV}, \xi_{i 1}=1.827, H_{i i}=-8 \mathrm{eV}, \xi_{i 1}=1.5$. Standard Hoffmann parameters were used for the $C$ and $H$ atoms.

16. Büttiker M., Imry Y., R. Landauer R. and Pinhas S., Phys. Rev. B 31, 6207 (1985).

17. Sellers H., Ulman A., Shnidman Y. and Eilers J.E., J. Am. Chem. Soc., 115, 9389 (1993).

18. Emberly E.G. and Kirczenow G. Phys. Rev. B 58, 10911(1998) 